\title{
SIGNIFICADOS Y PRÁCTICAS DOCENTES EN TORNO A LA EQUIDAD EN AULAS NORMALISTAS
}

\author{
MEANINGS AND TEACHING PRACTICES \\ REGARDING EQUITY IN \\ NORMALIST CLASSROOMS
}

\author{
María Guadalupe Loza Jiménez ${ }^{1}$ \\ https:// orcid.org/0000-0003-4106-5233 \\ Martha Leticia Gaeta González ${ }^{2}$ \\ https://orcid.org/0000-0003-1710-217X
}

Recibido: septiembre 21, 2020 - Aceptado: octubre 20, 2020

\section{RESUMEN}

La equidad en educación hace referencia a la capacidad de considerar la situación desigual de los alumnos y sus familias, de las comunidades y de las escuelas, así como de ofrecer apoyos especiales a quienes lo requieran, de tal forma que los objetivos educativos se alcancen por el mayor número de estudiantes. Uno de los principales impulsores de la equidad educativa son los docentes, por lo que es fundamental fortalecer la formación que les permita comprender la diversidad de alumnos y, llegado el caso, también a sus familias. La presente investigación tiene como objetivo describir los significados y las prácticas de profesores normalistas sobre la equidad, en la formación docente. La fundamentación teórica parte de la conceptualización de equidad en la Ética Nicomáquea de Aristóteles y se vincula con un ejercicio necesario en la educación actual. Se trata de un estudio cualitativo que, desde la investigación-acción, recoge el pensamiento de un grupo de once profesores de tiempo completo, integrantes de cuerpos académicos de una Escuela Normal del Estado de Puebla,

1 Escuela Normal «Instituto Jaime Torres Bodet». Maestra en Educación en Ciencias, Benemérita Universidad Autónoma de Puebla. Doctorante en Educación, Universidad Popular Autónoma del Estado de Puebla. Docente de tiempo completo, Escuela Normal «Instituto Jaime Torres Bodet». Integrante del Cuerpo Académico: IJTB-CA-1 Profesionalización Docente. Miembro de la Red de Investigadores de la Región Centro (REDIREC). Docente con Perfil PRODEP desde 2012. mariaguadalupe.loza@upaep.edu.mx

2 Doctora en Psicología y Aprendizaje, Universidad de Zaragoza, España. Docente de tiempo completo de la Facultad de Educación, Universidad Popular Autónoma del Estado de Puebla. Es miembro: del Sistema Nacional de Investigadores Nivel 1 (SNI-1); del Consejo Mexicano de Investigación Educativa (COMIE); de la Red Mexicana de Investigadores de la Investigación Educativa (REDMIIE); de la Red Nacional de Investigadores en Educación y Valores (REDUVAL) y de la Asociación Científica de Psicología y Educación, en España. marthaleticia.gaeta@upaep.mx 
México. A partir de una entrevista semiestructurada, los datos nos dejan ver que en su mayoría los profesores conocen el significado y asumen conductas para propiciar la equidad en el aula, aunque se requieren acciones concretas en colegiado para una práctica habitual de las mismas, que favorezca un desarrollo integral de los docentes en formación.

Palabras clave: equidad, equidad educativa, formación docente, educación normalista, actividades del profesor, educación superior.

\begin{abstract}
Equity in education refers to the ability to consider the unequal situation of students and their families, communities, and schools and offer special support to those who require it so that the largest number of students achieve educational objectives. One of the main drivers of educational equity is teachers, so it is essential to strengthening their training, allowing them to understand students' diversity and, if necessary, their families. This research aims to describe the meanings and practices of Normalist teachers on equity in teacher training. The theoretical foundation is based on the conceptualization of equity in Aristotle's Nicomachean Ethics and linked to a necessary exercise in current education. It is a qualitative study that, from action research, collects the thoughts of a group of eleven full-time teachers, members of academic bodies of a Normal school in the State of Puebla, Mexico. From a semi-structured interview, data show us that most teachers know the meaning and take on behaviors to promote equity in the classroom. However, concrete actions are required in collegiate for a regular practice, which favors teachers' integral development in training.
\end{abstract}

Key words: Equity, Educational Equity, Teacher Training, Normalist Education, Teacher Activities, Higher Education.

\title{
INTRODUCCIÓN
}

La educación equitativa, como se ha establecido en la Declaración Mundial sobre Educación para Todos (UNESCO, 1990), es aquella en donde la totalidad de la población puede satisfacer sus necesidades básicas de aprendizaje. Supone dotar a la población de herramientas esenciales como lectura, escritura y cálculo, así como el desarrollo de conocimientos teóricos y prácticos, valores y actitudes indispensables para que puedan vivir y trabajar con dignidad, participar en el desarrollo social, mejorar su calidad de vida, tomar decisiones fundamentadas y continuar aprendiendo.

En México, el artículo tercero constitucional menciona que toda persona tiene derecho a recibir educación; estableciendo como educación básica obligatoria los niveles de prescolar, primaria y secundaria. A su vez, la responsabilidad del Estado de proporcionar esta educación, queda recogido en la Ley General de Educación, estableciéndose que la equidad es un objetivo fundamental del sistema de enseñanza. Concretamente, el capítulo III, artículo 32 de esta Ley, indica que las autoridades educativas tomarán medidas tendientes a crear condiciones que permitan el ejercicio pleno del derecho a la educación de calidad de cada individuo, una mayor equidad educativa, así como el logro de la efectiva igualdad de oportunidades de acceso y permanencia a los sistemas educativos (Diario Oficial de la Federación, 2018). 
Por su parte, Rodríguez-Solera (2008) señala que la educación equitativa permite a todas las personas acceder a una educación básica de similar cantidad y calidad, con independencia de su origen socioeconómico, considerando como únicas diferencias aquellas requeridas para elevar el nivel académico de algunos grupos vulnerables, con necesidades educativas especiales o de rezago educativo. Estos elementos deben precisarse, por tanto, en la formación de los docentes, pues son ellos que atenderán a nuevas generaciones de individuos con características personales y contextuales diferentes que requieren de objetivos y metas específicas.

Corresponde a las autoridades implementar las condiciones para lograr una equidad educativa, estableciendo para los individuos las mismas oportunidades de acceso y obligatoriedad, y prestando los servicios educativos para que toda la población pueda cursar la educación básica. Sin embargo, dentro de las aulas es responsabilidad de los docentes implementar las estrategias y propuestas pedagógicas, desde las cuales abordar las prácticas educativas para atender las diferencias que presentan los estudiantes. Por ello, como menciona Amati (s/f), la formación docente es ineludible para mejorar la calidad de la educación con equidad, asumiendo las exigencias que ello conlleva.

Así, la formación profesional docente debe favorecer el desarrollo de competencias para la aplicación de la equidad como concepto de justicia en las aulas. Ello implica no solo el abordaje de contenidos curriculares, sino la formación integral que conlleve a la proyección de escenarios, más allá de lo determinado por las habilidades didácticas o instrumentales; es decir, precisa de la reconceptualización de la formación docente, concibiéndola como eje de oportunidad para el actuar emergente activo de las prácticas equitativas.

Cuando se reflexiona en la manera de acercar la equidad a los docentes en formación, es indiscutible pensar también en la forma en que ellos mismos son tratados por sus profesores dentro de las aulas; considerar entonces que los formadores de docentes deben tener el conocimiento y la experiencia para implementar estrategias y procesos de equidad con los estudiantes normalistas.

Ante esta premisa surgen las interrogantes: ¿qué significa para los formadores de docentes el término «equidad»?, ¿cuáles son las prácticas que realizan en las aulas en torno a ella?, ¿de qué manera lograr un acercamiento a las prácticas equitativas que propicien, en los futuros docentes, una educación más justa? Las respuestas a estas preguntas deben llevar a ejercicios de reflexión por parte de los formadores, que generen propuestas de cambio o reforzamiento de acciones para el logro de una formación docente con y en equidad.

Considerando que el profesorado es un elemento determinante en toda propuesta educativa, el objetivo de la presente investigación se centra en describir los significados y las prácticas que, en torno a la equidad, realizan los formadores de docentes en las aulas de una Escuela Normal del estado de Puebla, México.

\section{REFERENTES TEÓRICOS}

\section{Definición de equidad}

Hablar de equidad implica remitirnos a la Ética Nicomáquea, cuyo autor nos revela que: 
[...] lo equitativo, en efecto, siendo mejor que cierta justicia, es justo; y por otra parte, es mejor que lo justo no porque sea de otro género. Por tanto, lo justo y lo equitativo son lo mismo, y siendo ambos buenos, es, con todo, superior lo equitativo (Gómez-Robledo, 2016: 96).

A través del tiempo se ha identificado cierta dificultad para comprender que lo equitativo realmente es justo, pero no ante la ley, es decir, no es posible promulgar correctamente una disposición en general.

[...] cuando la ley hablare en general y sucediere algo en una circunstancia fuera de lo general, se procederá rectamente corrigiendo la omisión en aquella parte en que el legislador faltó y erró por haber hablado en términos absolutos, porque si el legislador mismo estuviera presente, así lo habría declarado, y de haberlo sabido, así lo habría legislado (Gómez-Robledo, 2016: 96).

Quien emite las leyes, establece pautas de conducta universales con la finalidad de que exista una estabilidad sociopolítica, ya que cada miembro de esa sociedad debe atenerse a un mismo criterio de conducta en cada caso. Las normas toman en cuenta las situaciones conocidas, y de estas, las que surgen con mayor frecuencia, es decir, las que son típicas.

La Ética Nicomáquea advierte que ese tratamiento singular de aquellos casos no previstos por las reglas generales, en aras de mantener el estándar de justicia alcanzado con dichas reglas para los casos típicos es la equidad, es decir, se trata de la justicia para la consideración de los casos atípicos. En palabras del propio Aristóteles, la equidad no es menos que la justicia, sino que es la justicia misma operada en casos no previstos por esta. Equidad es un término que en muchas ocasiones se ha considerado sinónimo de «igualdad»; ambos son valores que se piensan a partir de aquello considerado desigual o inequitativo (Sen, 1992). Es por ello que un gran número de trabajos investigativos sostienen una diferencia conceptual entre equidad e igualdad. La igualdad y la libertad constituyen dos importantes pilares de las naciones occidentales contemporáneas. A diferencia de las sociedades estamentales -en las que se reconoce la existencia de grupos o castas con distintos derechos-, las sociedades «abiertas» parten de la idea de igualdad ante la ley y suponen la existencia de ciudadanos que ejercen deberes y derechos en un contexto de libertades civiles, políticas y económicas (Rodríguez-Solera, 2008).

Según refiere Calvo (2013), la equidad parte del reconocimiento de las desigualdades intrínsecas de los sujetos, en diversas dimensiones de la vida social. Nos encontramos ante un término de gran trascendencia para las relaciones humanas, ya que la equidad integra a la igualdad y la amplía en sus múltiples dimensiones. Así, «la noción de equidad renuncia a la idea de que todos somos iguales y a partir del reconocimiento de las diferencias, propone estrategias para lograr la igualdad fundamental que debería aportar la educación básica» (Calvo, Camargo y Gutiérrez, 2006: 14).

Para Rawl, (2002) las desigualdades socioeconómicas, han de satisfacer dos condiciones: primero, estar asociadas a cargos y posiciones abiertas a todos, en las condiciones de una equitativa igualdad de oportunidades; y segundo, procurar el máximo beneficio de los miembros menos aventajados de la sociedad.

Aunque sabemos que no existe una sociedad por completo igualitaria, sí puede aspirarse a una sociedad equitativa, en la cual -si bien seguirán existiendo infinidad de desigualdades- se promueva la igualdad como un aspecto fundamental para construir una sociedad justa (Rodríguez-Solera, 2008). 
En el ámbito de la economía, el concepto de equidad no tiene el significado de moralidad, legalidad o justicia que se le otorga en otros escenarios; su objetivo final es satisfacer las necesidades del ser humano (Vergara, 2005). A este respecto, el autor menciona que:

[...] el ser humano no solo debería tener la libertad de escoger un trabajo de acuerdo a su aptitud y a las oportunidades que se le presenten, sino también sobre la base de las ventajas y desventajas que ese trabajo le ofrezca, siempre y cuando se dé en una economía libre, que tendería a ser más o menos equitativa, pero si no fuera así, la libertad que cada persona tiene se vería muy limitada por las oportunidades que esa sociedad ofrezca (p. 187).

Para los socialistas, una sociedad equitativa promueve la igualdad económica, aun a costa de otras libertades fundamentales, de tal forma que lo importante son los resultados económicos; mientras que para los liberales, una sociedad equitativa garantiza la libertad e igualdad de oportunidades, incluso cuando su funcionamiento implique la desigual distribución de la riqueza o de otros objetos sociales valiosos (Canto, 2015).

\section{Equidad educativa}

La educación de los pueblos siempre ha buscado la mejora. Sin embargo, la mejora en la educación es un tema controvertido. Según Escudero, González y Rodríguez (2013), habría que remitirse a valores como igualdad y libertad, democracia y justicia social y educativa, inclusión y participación, todos ellos con significados, interpretaciones e implicaciones diferentes. Para el autor, la equidad tiene una larga historia, «recientemente se aprecia un renovado foco en la misma y sus implicaciones para el currículo y la enseñanza, la organización y el liderazgo escolar, así como respecto de las administración y políticas sociales y escolares» (p. 209).

En el ámbito de la educación, el concepto de equidad se vincula con el de calidad y son muchas las variables que componen, describen y explican la condición sociológica de lo que, en los últimos años, han llamado equidad educativa también relacionada con conceptos tradicionales como igualdad de oportunidades y justicia social o eficiencia (Lara-Villanueva, 2010). En este mismo sentido, el concepto se asocia al de cobertura, es decir que a los individuos se les otorguen beneficios que contribuyan a mejorar sus condiciones de vida.

La Comisión Económica para América Latina (CEPAL), reconoce dos variables necesarias para dar respuestas equitativas a la población por parte del Estado, con un carácter tutelar. Se denomina equidad horizontal al trato igualitario a personas en condiciones similares y que justifican el gasto en educación primaria; mientras que la equidad vertical es el trato diferenciado a individuos que lo requieran (Flood, 1994).

La equidad debe analizarse desde diversos ángulos, ya que no solo se refiere a la distribución de la educación en un país, sino también a la identificación de desigualdades que en un aula pueden tener los individuos, o a las distintas categorías u oportunidades que presentan. Para estudiar la equidad en educación se deben conocer las diferencias en el logro educativo, entre los individuos; las oportunidades entre grupos (etnias, género, grupos de edad, entre otros); el contexto socioeconómico y cultural e, incluso, la reproducción de desigualdades o la sustitución de estas por otras más. 
Con base en lo expuesto por Bracho (2010: 9), en educación, equidad implica: «Educar de acuerdo con las diferencias y necesidades individuales, sin que las condiciones económicas, demográficas, geográficas, éticas o de género supongan un impedimento al aprendizaje». Se refiere a tomar en cuenta la situación desigual de los alumnos y sus familias, de las comunidades y las escuelas, ofrecer apoyos especiales a quienes lo requieren, de forma que los objetivos educativos sean alcanzados por el mayor número de estudiantes.

Para Martínez y Dijk (2020: 80), la equidad educativa se entiende como «el mecanismo que posibilita el mismo horizonte de oportunidad para todos los estudiantes de un sistema educativo, sin importar su procedencia socioeconómica». Esta definición la exponen después de analizar que, en México, se espera que los estudiantes tengan los mismos resultados de desempeño, sin considerar que provienen de distintos niveles socioeconómicos.

Otro de los términos vinculado con el concepto de equidad es el de inclusión educativa, que en las últimas décadas y a nivel mundial se ha hecho presente en los discursos político-pedagógicos en un contexto en el que, como menciona Rodríguez-Solera (2008), simultáneamente se profundizan por procesos de exclusión y se reconocen las necesidades institucionales y pedagógicas para lograr las metas de una educación de calidad para todos. El mismo autor define a la educación inclusiva como el proceso para tratar de garantizar el aprendizaje y la participación de todos los estudiantes en la vida escolar de las instituciones educativas, con particular atención a aquellos niños y jóvenes vulnerables.

El Modelo Educativo 2016 (SEP, 2016) hace referencia a la equidad y a la inclusión como principios fundamentales para el funcionamiento del sistema educativo mexicano. En dicho documento se menciona que:

\footnotetext{
Esto implica velar porque diversos elementos que son parte o influyen en su operación, observen estos principios de manera transversal: normatividad, infraestructura, presupuesto, becas, valores y actitudes, planes, programas, métodos y materiales, ambiente escolar y prácticas educativas, gestión escolar, evaluación, capacitación, sistemas de información, maestros, directores, supervisores, padres y madres de familia (SEP, 2016: 66).
}

Si bien es cierto que este modelo se orienta a la educación básica y media superior, el planteamiento es aplicable en el contexto de la formación docente, ya que hace énfasis en crear las condiciones para garantizar el acceso efectivo a una educación de calidad, reconociendo las diferencias individuales de los estudiantes y atendiendo las necesidades diversificadas que contribuyan a eliminar las barreras que limitan el acceso a la educación, el aprendizaje y la participación.

Sin embargo, como menciona García (2018: 56), en el modelo se plantean afirmaciones generales sobre los propósitos de la inclusión, pero «no se señala cómo se puede poner en marcha en las aulas, ni se define la política que se seguirá, ni la evidencia en que se basará dicha política».

\section{Equidad en la formación docente}

En el ámbito de las profesiones educativas, un aspecto relevante en la formación docente $-y$, a partir de ella, la forma en que los futuros profesores conciben la equidad, igualdad e inclusión- es cómo llevar a la práctica estas nociones durante el trayecto formativo en la Escuela Normal. Sin duda alguna, como menciona Rodríguez-Solera (2008), el futuro educador replica aquellos modelos pedagógicos y didácticos en los que se formó; si se desean docentes 
formados para la equidad, igualdad e inclusión, deben estar expuestos a prácticas pedagógicas y didácticas que las potencien, desde la formación inicial.

Un docente formado para la equidad y la inclusión debe contar con una amplia y sólida formación pedagógica (Calvo, 2013). Es por ello que los postulados de la pedagogía activa -además del conocimiento de los postulados de la pedagogía crítica-, pueden ser de gran utilidad para contar con las competencias que flexibilicen los espacios de enseñanza y de aprendizaje, y que formen partiendo de propuestas que reconozcan las capacidades cognitivas y expresivas de los estudiantes.

El conocimiento y uso de estrategias didácticas que se acercan con la mejor metodología a las condiciones estudiantiles, es sin duda un factor importante para el diagnóstico y manejo de las diferencias en el aula. Algunas formas apropiadas para hacerlo, como afirma Martínez-Otero (2011), van desde la disposición de más tiempo para cada alumno, hasta la personalización del proceso, pasando por reconocer sus saberes previos y exponiendo distintas formas de enseñar y diferentes formas para aprender.

El docente que pretende atender con equidad a los estudiantes, podrá hacerlo de mejor manera mediante el fortalecimiento de su identidad profesional y ética, lo cual le exige enfatizar su compromiso social y que cuente con el desarrollo de las competencias para la vida. Debe desempeñarse en el aula, promoviendo prácticas de congruencia entre lo que versa y lo que actúa, atendiendo a los estudiantes con base en sus particularidades. De acuerdo con Lara-Villanueva (2010), el concepto de aulas equitativas implica valorar a la persona con derechos y necesidades:

Dentro de las aulas debe trabajarse desde la situación social y académica que se presenta, porque cada realidad educativa es diferente, por lo tanto, ser equitativo significa también que deben valorarse las capacidades de los niños y las niñas, aun cuando estos provengan de entornos socioculturales y económicos diferentes (p. 9).

Todo ello, considerando dos dimensiones importantes; lo individual y lo social (como grupo). De modo que la formación de un profesional de la educación es un camino largo que comienza con la formación inicial, y se desarrolla y fortalece en la práctica con apoyo de sus pares, autoridades inmediatas y con procesos de capacitación acordes con sus necesidades específicas y evaluaciones pertinentes (Compromiso Social, 2017).

Para realizar prácticas con equidad es indispensable, por tanto, que el docente conozca las normas, los derechos de los niños, de los jóvenes, de las mujeres, de todos los ciudadanos; que los respete, fomente y que los haga valer dentro y fuera de las aulas, teniendo presente que entre más avanzada es la propuesta educativa, su papel se vuelve más complejo, pues debe dejar que el estudiante camine por sí mismo, pero acompañando, interviniendo y proponiendo en la justa medida.

\section{METODOLOGÍA}

La presente investigación se aborda con un enfoque cualitativo que, desde la hermenéutica, parte del supuesto que los actores sociales no son meros objetos de estudio, sino que también significan, hablan, pueden ser observados como subjetividades que deciden y son capaces de reflexionar sobre su situación (Monje-Álvarez, 2011). 
En este estudio, la forma de construir el conocimiento se centra en la investigación-acción que, de acuerdo con Martínez (2014), representa un proceso mediante el cual los sujetos investigados son auténticos coinvestigadores, participando activamente en el planteamiento del problema (que es algo que les afecta e interesa), en la información que debe obtenerse al respecto, en los métodos y las técnicas que se utilizarán, en el análisis e interpretación de los datos, en la decisión de qué hacer con los resultados y qué acciones se programarán para su futuro.

\section{Participantes}

Participaron en este estudio once docentes, profesores de tiempo completo (PTC), que pertenecen a Cuerpos Académicos (CA) de una Escuela Normal y atienden programas educativos de las licenciaturas en Educación Preescolar y en Educación Secundaria. Este número se determinó a partir del tipo de estudio, buscando reflejar las opiniones de los profesores de manera profunda.

Tabla 1. Datos sociodemográficos de los docentes participantes, LGAC y asignaturas que atienden

\begin{tabular}{|c|c|c|c|c|c|c|c|}
\hline 节 & 票 & : & $\begin{array}{l}\text { 岀 } \\
\text { 这 }\end{array}$ & 可 & $\begin{array}{l}\text { U } \\
\text { ṫं }\end{array}$ & $\begin{array}{l}\text { Línea de generación } \\
\text { y aplicación del } \\
\text { conocimiento que cultiva }\end{array}$ & $\begin{array}{c}\text { Asignaturas impartidas en los } \\
\text { últimos dos años }\end{array}$ \\
\hline D1 & 46 & M & 15 & 15 & 3 & $\begin{array}{l}\text { La tecnología en la práctica } \\
\text { educativa. }\end{array}$ & $\begin{array}{l}\text { Plano cartesiano y funciones/ } \\
\text { Observación y práctica } \\
\text { docente/ Tecnología y } \\
\text { didáctica de las matemáticas. }\end{array}$ \\
\hline D2 & 57 & H & 14 & 9 & 3 & Innovación educativa / TIC. & $\begin{array}{l}\text { Medición y cálculo } \\
\text { geométrico/ Observación y } \\
\text { práctica docente/ Introducción } \\
\text { a la enseñanza de las } \\
\text { matemáticas. }\end{array}$ \\
\hline D3 & 49 & $\mathrm{H}$ & 14 & 9 & 2 & $\begin{array}{l}\text { Enseñanza-aprendizaje de } \\
\text { las matemáticas. }\end{array}$ & $\begin{array}{l}\text { Observación y práctica } \\
\text { docente/ Seminario de temas } \\
\text { de historia de las matemáticas/ } \\
\text { Pensamiento algebraico. }\end{array}$ \\
\hline D4 & 43 & M & 20 & 20 & 1 & Prácticas pedagógicas. & $\begin{array}{l}\text { Estrategias de intervención } \\
\text { educativa/ Innovación } \\
\text { educativa/ Estrategias de } \\
\text { enseñanza y aprendizaje. }\end{array}$ \\
\hline D5 & 62 & M & 35 & 23 & 7 & $\begin{array}{l}\text { Competencias docentes. } \\
\text { Educación ambiental. }\end{array}$ & $\begin{array}{l}\text { Educación ambiental para } \\
\text { la sustentabilidad/ Trabajo } \\
\text { docente/ Taller de diseño } \\
\text { de propuestas didácticas y } \\
\text { análisis del trabajo docente. }\end{array}$ \\
\hline
\end{tabular}




\begin{tabular}{|l|l|l|l|l|l|l|l|}
\hline D6 & 44 & M & 19 & 19 & 7 & Competencias docentes. & $\begin{array}{l}\text { Estrategias de trabajo docente/ } \\
\text { Desarrollo socioemocional del } \\
\text { niño/ Adquisición de la lengua } \\
\text { materna. }\end{array}$ \\
\hline D7 & 55 & M & 19 & 19 & 7 & $\begin{array}{l}\text { Colegialidad. } \\
\text { Investigación de la práctica } \\
\text { educativa. }\end{array}$ & $\begin{array}{l}\text { Trabajo docente/ Taller } \\
\text { de diseño de propuestas } \\
\text { didácticas y análisis del } \\
\text { trabajo docente/ Planeación } \\
\text { educativa. }\end{array}$ \\
\hline D8 & 41 & M & 11 & 11 & 7 & Trayectorias estudiantiles. & $\begin{array}{l}\text { Producción de textos } \\
\text { académicos/ Adecuación } \\
\text { curricular/ Gestión educativa. }\end{array}$ \\
\hline D9 & 51 & H & 34 & 15 & 3 & $\begin{array}{l}\text { Procesos de formación y } \\
\text { prácticas educativas. } \\
\text { Alfabetización académica. }\end{array}$ & $\begin{array}{l}\text { Trabajo docente/ Taller } \\
\text { de diseño de propuestas } \\
\text { didácticas y análisis del } \\
\text { trabajo docente/ Procesos } \\
\text { cognitivos y cambio } \\
\text { conceptual en matemáticas. }\end{array}$ \\
\hline D10 & 44 & M & 19 & 19 & 5 & $\begin{array}{l}\text { Elaboración de documento } \\
\text { recepcional. } \\
\text { Concepto de variable. }\end{array}$ & $\begin{array}{l}\text { Pensamiento algebraico/ } \\
\text { Seminario de investigación en } \\
\text { educación matemática/ } \\
\text { Taller de diseño de propuestas } \\
\text { didácticas y análisis del } \\
\text { trabajo docente. }\end{array}$ \\
\hline 35 & M & 10 & 10 & 3 & $\begin{array}{l}\text { Estrategias de aprendizaje. } \\
\text { Procesos cognitivos/ } \\
\text { Presentación y tratamiento de } \\
\text { la información/ Observación y } \\
\text { práctica docente. }\end{array}$ \\
\hline
\end{tabular}

\section{Técnicas de recolección de datos}

Se realizaron entrevistas semiestructuradas, mediante la utilización de una guía con diez preguntas, iniciando por una ficha de identificación cuyos datos comprendían: edad, género, años de servicio en educación y años de servicio en la Escuela Normal. En seguida se interrogó acerca de las líneas de generación y aplicación de conocimiento que se cultivan (LGAC) en el Cuerpo Académico y las asignaturas que han impartido en los últimos dos años. La última parte del instrumento se centró en el significado del término «equidad»y en las prácticas realizadas para favorecer la equidad dentro del aula. En caso de una respuesta negativa, se interrogó la causa y en caso de una respuesta afirmativa, se solicitó que el participante relatara de qué forma lo realizaba. 


\section{Procedimiento de recolección de datos}

Con la autorización de los directivos institucionales, se tuvo un acercamiento a los docentes participantes para solicitar su consentimiento informado. Asimismo, se les comunicó el objetivo de la investigación, sus alcances y posibles lugares de difusión. Se solicitó la autorización para grabar su voz durante la entrevista, y su anuencia para publicar los comentarios emitidos, sin que ello implicara revelar la fuente, y señalar específicamente la institución de referencia. Las entrevistas a los participantes se realizaron de manera individual, con una duración promedio de 20 minutos cada una, mismas que fueron audio grabadas y posteriormente transcritas para su análisis.

\section{Análisis de los datos}

La información obtenida de las entrevistas (por medio de audio grabación), se transcribió de manera digital en un formato que permitiera la categorización y las anotaciones especiales, numerando las páginas y las líneas del texto para facilitar su referencia. Se realizó una lectura global y repetitiva de los textos, tratando de obtener una visión general de toda la información recolectada. Con base en la propuesta de Martínez Miguélez (2012), se dividieron los contenidos en porciones (párrafo o grupos de párrafos que expresan una idea o un concepto central), logrando identificar las ideas principales y hacer anotaciones al margen que permitieran tipificarlas; se identificaron los patrones comunes y se señalaron elementos similares.

Para los fenómenos sociales, Lofland (citado por Martínez Miguélez, 2012: 75) sugiere seis categorías: actos, actividades, significados, participación, relación y situaciones y, para los fines de la presente investigación, se han considerado tres: a) Actividades: acciones de mayor duración (días, semanas, meses) que involucran a las personas de forma significativa; b) significado: expresiones verbales de los participantes que definen y dirigen su acción, y c) participación: compromisos que involucran plenamente a las personas en la situación estudiada. Con base en ello, se tomaron en cuenta actividades que realizan los profesores en las aulas para fomentar la equidad, el significado que ellas expresan y la forma en la que se involucran en la práctica docente.

\section{Consideraciones éticas}

La presente investigación sustenta una postura ética con el manejo de los datos recabados y la participación de los sujetos. Se realizaron las solicitudes pertinentes a las autoridades de la institución participante. Una vez comprendida la finalidad y alcance de la investigación por parte de los docentes, y en caso de estar de acuerdo, se les solicitó consentimiento por escrito para dar cuenta de su participación voluntaria y desinteresada.

Los datos obtenidos por medio de las entrevistas han sido resguardados y empleados exclusivamente para los fines de la investigación: no están disponibles para ningún otro propósito u otra persona. La institución y los participantes han sido nominados y referidos de manera que no se evidencien los datos que les identifiquen directamente. 


\section{RESULTADOS}

Los once docentes participantes en la presente investigación (como se mostró en la tabla 1), son tres hombres y ocho mujeres, con edades entre 35 y 62 años. Su trayectoria docente varía entre diez y 35 años, y su experiencia en educación normalista va desde los diez hasta los 23 años. Pertenecen a los cuatro cuerpos académicos de la institución con un registro ante el Programa de Mejoramiento del Profesorado (PRODEP), de tres a siete años. Al momento del presente estudio, cinco de ellos atendían el programa educativo de la Licenciatura en Educación Secundaria con especialidad en Matemáticas y uno con especialidad en Biología. Los otros cinco atendían el Programa de la Licenciatura en Educación Preescolar. Los profesores cultivan líneas de generación y aplicación del conocimiento (LGAC) en relación con: innovación educativa; prácticas pedagógicas; prácticas educativas; procesos de formación y prácticas educativas; procesos de enseñanza y aprendizaje de las matemáticas; tecnología en la práctica educativa; competencias docentes; estrategias de aprendizaje; alfabetización académica; trayectorias estudiantiles; educación ambiental en la formación docente; colegialidad e investigación de la práctica educativa.

Las asignaturas que imparten se relacionan con los campos de formación específica (matemáticas y preescolar) y con la línea de acercamiento a la práctica escolar (observación y práctica docente, trabajo docente y taller de diseño de propuestas didácticas, y análisis del trabajo docente).

Gracias a la información obtenida puede identificarse que los docentes, en su mayoría mujeres, tienen una antigüedad significativa en el ámbito educativo y específicamente en la formación docente. Ello hace suponer que en algún momento de su trayectoria pudieron exponerse a situaciones de inequidad o desigualdad, lo que a su vez implica que de cierto modo han tenido experiencia en el manejo de estas.

El ser integrante de un cuerpo académico reconocido por el PRODEP requiere cierto perfil profesional, con experiencia en docencia, investigación, gestión, dirección de tesis y tutoría, además del cultivo de ciertas LGAC. En relación con estas últimas, es de llamar la atención que de las 16 líneas individuales establecidas para el desarrollo de procesos educativos en la institución, ninguna se orienta directamente al abordaje de equidad, igualdad o inclusión en la Escuela Normal.

Otro aspecto que se evidenció es que las asignaturas que imparten los participantes no precisan acciones concretas para el manejo de la equidad; los contenidos que se abordan están más en relación con la formación específica, pero ello no es impedimento para realizar una práctica educativa que favorezca la equidad en el aula.

Respecto del significado que cada uno otorga al termino equidad, en cinco casos se vincula con «la igualdad» $\mathrm{y}$, en los seis casos restantes, coinciden en ideas como: proporcionar a cada quien lo que necesita; brindar más apoyo a quien más lo requiere, y apoyar a sujetos o grupos humanos según sus circunstancias y las características o condiciones que cada uno tiene. 
Tabla 2. Significado que los formadores de docentes otorgan al termino equidad

\begin{tabular}{|c|c|}
\hline Docente & Significado de equidad \\
\hline D1 & ... equidad significa igualdad. \\
\hline D2 & Igualdad. \\
\hline D3 & Igualdad. \\
\hline D4 & ... darles a todos iguales oportunidades. \\
\hline D5 & ... establecer relaciones de igualdad. \\
\hline D6 & ... dar más apoyo a quien más lo requiere. \\
\hline D7 & ... darle a cada quien lo que necesita. \\
\hline D8 & ... que a cada quien se le dé lo que necesita. \\
\hline D9 & $\begin{array}{l}\text {... apoyar a sujetos o grupos humanos según las necesidades y } \\
\text { características que cada uno tiene. }\end{array}$ \\
\hline D10 & $\begin{array}{l}\text {... proporcionar lo que los alumnos o personas necesiten... no } \\
\text { necesariamente que sea igual para todos. }\end{array}$ \\
\hline D11 & Dar a cada quien lo que necesite de acuerdo a sus circunstancias. \\
\hline
\end{tabular}

Tal pareciera que otorgar significado a un término resulta fácil; muchas de las veces ese término es tan usual, tan común, de un lenguaje cotidiano en el ámbito educativo, que pasa desapercibido. Para algunos de estos formadores de docentes, si bien no es muy claro el significado, se acerca a una condición semejante. Para otros, el término adquiere una connotación apropiada en virtud de brindar apoyo a quienes más lo requieren con base en una condición específica. Sin embargo también es posible identificar que en la mitad de los participantes, el significado se relaciona estrechamente a la equidad de género; cuatro más la vinculan con necesidades psicoafectivas; seis con aspectos cognitivos y de aprendizaje, y solo dos hacen referencia a aspectos económicos. Cabe señalar que en algunos casos, los docentes mencionaron más de un aspecto de la equidad.

En relación con las prácticas descritas por los profesores en torno a la equidad dentro del aula, pueden clasificarse como:

a) Realización de diagnósticos de necesidades estudiantiles.

b) Trato igualitario.

c) Implementación de estrategias didácticas diversificadas.

d) Implementación de actividades tutoriales, individuales o colectivas. 
e) Impartición de asesorías académicas individuales.

f) Apoyo en situaciones específicas (madres solteras, dificultades económicas, etcétera).

Todas ellas se expresan a continuación:

a) Respecto de las prácticas de equidad como parte del diagnóstico de necesidades estudiantiles, algunos docentes afirman, por ejemplo:

D7. [...] diversificando estrategias para las diferencias en el aprendizaje, tanto en los estilos como en los ritmos, por supuesto haciendo un diagnóstico que permita darme cuenta de esas diferencias, y también siendo tolerante ante ciertas circunstancias relacionadas con las diferencias socioeconómicas.

D11. [...] considero que sí, al atender las necesidades de mis estudiantes de acuerdo a su condición, al conocer al menos de manera general su contexto familiar y sus necesidades académicas.

Realizar prácticas de diagnóstico para identificar desigualdades y permitir un trato equitativo supone para los docentes la diversificación de actividades, acordes con las necesidades y condiciones particulares de cada estudiante. Como mencionan Escudero et al. (2013), es una actitud vigilante de prevención y respuestas a los riesgos de exclusión que afectan desmesuradamente a los más desprotegidos: «De manera que desde aquí reside la elaboración de una agenda de las mejoras por acometer» (p. 218).

b) En lo que respecta al trato igualitario, algunos docentes refieren:

D1. [...] les doy un trato igualitario en actividades y todo, sin distinción de sexos.

D2. [...] las actividades que les pongo son iguales, sin distinción de género, y la forma en la que les hablo es igual para todos.

D3. [...] distribuyo actividades iguales para hombres y mujeres.

D5. [...] mediante estrategias organizadas para desempeñarse de manera justa, sin tomar en cuenta estereotipos, prejuicios derivados de actitudes machistas o feministas.

Con estas afirmaciones observamos que los docentes otorgan un trato igualitario en relación con el género. Las actividades que promueven son las mismas para mujeres y hombres, no existe discriminación por tal característica; se ofrecen las mismas oportunidades y los mismos beneficios y, como deja ver uno de ellos, se cuida el aspecto del desempeño justo, dando así el sentido de equidad, con el que se atiende a la idea de que hombres y mujeres son distintos, pero se les ofrecen las mismas oportunidades basadas en características contextuales y necesidades específicas.

Estos testimonios son congruentes con los planteamientos de la «Ley General para la igualdad entre hombres y mujeres», publicada en el Diario Oficial de la Federación, el 2 de agosto de 2006, la cual ha tenido hasta el momento más de siete reformas (OIT, 2015; DOF, 2018), y que define igualdad de género como:

Situación en la cual mujeres y hombres acceden con las mismas posibilidades y oportunidades al uso, control y beneficio de bienes, servicios y recursos de la sociedad, así como a la toma de decisiones en todos los ámbitos de la vida social, económica, política, cultural y familiar (p. 2). 
Los mensajes que continuamente se reciben a través de comunicados oficiales, redes sociales y medios de información, llevan a los docentes a actuar considerando las distintas identidades y voces presentes en las aulas y, como menciona Valdivielso (2016: 8), los obligan a «escuchar las demandas de reconocimiento de los estudiantes y a dar respuestas dirigidas para garantizar en ellos la igualdad de oportunidades, de condiciones y de trato».

La implementación de estrategias didácticas diversificadas son expresadas como:

D4. [...] les pongo ejercicios reflexivos para empatizar con diferentes necesidades de aprendizaje y que sean solidarias con sus compañeras. Creo que sí funciona.

D9. [...] hay alumnos con necesidades específicas de apoyo, por ejemplo, cuando entregan un trabajo con ciertas características, por ejemplo, un mapa conceptual y a los estudiantes que se les detecta con productos con lo no requerido, se identifican las causas y se trabaja con él.

D10. [...] atendiendo necesidades académicas, algunas características de sus competencias o al evaluar.

Advertimos en estos testimonios de docentes normalistas, como lo menciona Cruz Vadillo (2019: 2): «[que] atienden a cuestiones didáctico-pedagógicas, pretendiendo innovar las prácticas educativas con la incorporación de nuevos principios metodológicos que impliquen la posibilidad de atender a la diversidad áulica [...]».

En la conceptualización de aulas equitativas, Lara-Villanueva (2010) señala que no deben dejarse de lado las estrategias didácticas con que el docente puede trabajar; en ellas debe promoverse la interacción social como base de los procesos escolares, lo que conlleva a la toma de decisiones para planificar estrategias de aprendizaje, como el trabajo cooperativo y el aprendizaje compartido. El enfoque del empleo de estas estrategias, pretende que los estudiantes sean protagonistas de su proceso formativo y, al mismo tiempo, beneficie a los compañeros de clase a mediar positivamente sus competencias académicas y a enriquecer sus procesos personales y sociales.

Para el docente, promover la equidad en el aula implica un diseño y una planificación de estrategias didácticas para atender dos aspectos importantes que pueden afectar las relaciones interpersonales: acceso al conocimiento, intercambio social, cognitivo y afectivo, así como establecimiento de relaciones justas (aceptación y reconocimiento del otro con sus diferencias), con lo cual se espera que el estudiante logre aprender (Lara-Villanueva, 2010: 11).

c) La implementación de actividades tutoriales, ya sea individuales o colectivas, son expresadas como:

D8. [...] mediante la tutoría con apoyos en el ámbito académico, administrativo y en el afectivo.

En consonancia con este testimonio cabe señalar que, en las Escuelas Normales, los docentes que realizan procesos de tutoría lo hacen bajo una estrategia institucional, mediante la cual brindan un acompañamiento personalizado a los estudiantes para orientarlos en la toma de decisiones y apoyarlos en las dificultades que pueden presentar a lo largo de su trayectoria escolar (Merino, 2018).

De esta forma, las actividades que realiza el docente como tutor, implican un ejercicio sustentado en una concepción humanista, buscando el desarrollo del estudiante bajo un marco de valores. En este sentido, la diversidad cobra gran importancia, y el reconocimiento y atención de las diferentes capacidades, intereses y motivaciones requiere de un trato 
equitativo y justo. Para ello es necesario, como lo mencionan Cintora, González y Vargas (2019: 8): «capacitar a los tutores en el desarrollo de estrategias diversificadas de enseñanza que les permitan responder a la diversidad en el aula».

Estas prácticas se alinean al concepto de tutoría formulado por la UNESCO (Romo, 2011: 48), señalando que: «comprende un conjunto de actividades que propician situaciones de aprendizaje y apoyan el buen desarrollo del proceso académico, con el fin de que los estudiantes, orientados y motivados, desarrollen automáticamente su propio proceso».

d) En cuanto a la impartición de asesorías académicas individuales, como señala uno de los profesores:

D6. [...] ofreciendo más apoyo a quienes lo requieren a través de asesoría, de mayor tolerancia en tiempos de entrega, más revisiones a los trabajos, supervisión de sus prácticas, también a través de la orientación educativa con asesorías.

En su mayoría, los docentes consideran que ante la heterogeneidad presentada en las aulas, es importante adecuar el desarrollo de las actividades a las necesidades propias de los estudiantes. En concordancia con Lara-Villanueva (2010), ser equitativo significa que deben valorarse y reconocerse las capacidades con las que cuentan los estudiantes y trabajar potenciando sus capacidades cognitivas y sociales.

$\mathrm{Al}$ ofrecer este tipo de actividades, el docente normalista vincula la equidad con otros valores: compromiso, responsabilidad, esfuerzo, diálogo, amistad, paz, entre muchos otros que, como formador de formadores, debe expresar de manera congruente y honesta.

e) Respecto del apoyo en situaciones específicas (madres solteras, dificultades económicas, etcétera), uno de los profesores menciona:

D9. [...] Hay que dar atención y asesoría de acuerdo con sus necesidades, por ejemplo, en el caso de las estudiantes que son madres, por lo que por salud del bebé no pueden estar, se ofrecen alternativas para que no afecten sus resultados académicos, o cuando hay intercambio para socializar temas dentro del aula, se sugiere cierta ayuda a algunos estudiantes.

Es importante señalar que la condición de madre y estudiante es una situación cada vez más frecuente en la Escuela Normal. Esta circunstancia marca una diferencia que la mayoría de las veces repercute en el ámbito escolar, pues como lo menciona Miller (2016), las condiciones, la multiplicidad de roles y la generación de vínculos al interior de los espacios educativos, les llevan a generar estrategias diversas que coadyuvan en la permanencia de la educación.

En tal sentido, si bien los docentes pueden considerar como normativas las políticas de inclusión emitidas desde las instituciones gubernamentales para este tipo de estudiantes, también observan la necesidad de implementar estrategias en el aula, para atender las características diferenciadas de las docentes en formación con hijos, sus necesidades y condiciones, logrando con ello la puesta en marcha de verdaderas prácticas de equidad en el aula. 


\section{CONCLUSIONES}

A través de este estudio, puede afirmarse que hablar de equidad educativa es un tanto complejo, cuando por principio se refiere a la atención de ciertas desigualdades, que la mayoría de las veces exponen situaciones de vulnerabilidad. Es considerar, como menciona Bracho (2010), aquellos casos atípicos que estando fuera de la institución, requieren atenderse.

Como hemos analizado, equidad e igualdad son conceptos ampliamente vinculados, aun cuando el de equidad es más comprensivo, ya que incluye un valor moral o ético para que un bien o servicio no sea distribuido de forma igualitaria, sino en beneficio de los factores o individuos menos favorecidos (Calvo, 2013; Calvo et al., 2006). La equidad se relaciona con la justicia, bajo el argumento de que la distribución de los bienes debe realizarse de acuerdo con lo que cada quien merece. La justicia a su vez se sustenta en el derecho, de modo que, ya que todos son iguales ante la ley, nadie debe ser privado de lo que por derecho le corresponde. De esta forma, la justicia utiliza como criterio práctico único la igualdad para regular la relación entre lo desigual.

Como indicamos, en educación la igualdad se justifica cuando se refiere a sujetos iguales en dignidad, siendo activos de iguales derechos y libertades, y teniendo las mismas oportunidades. Pero este concepto es inadecuado si se emplea como sinónimo de uniformidad y homogeneidad para realidades concretas, procesos o acciones.

Coincidimos con Calvo (2013) en que la formación docente requiere de una sólida formación pedagógica para generar condiciones de igualdad en los individuos, a través de la equidad y la inclusión. Es por tanto que en las Escuelas Normales las prácticas equitativas se deben vivir al interior de las aulas y en los contextos inmediatos, ya que corresponde a los formadores de docentes implementar acciones vivenciales que generen, a su vez, nuevas formas de trato equitativo.

A partir de la presente investigación, se concluye que la mayoría de los docentes participantes asumen significados y prácticas equitativas acordes con la justicia y la búsqueda de la igualdad; y aunque aún existe cierta distancia entre la intención y la acción, se vislumbra un actuar en solitario para la atención de las necesidades estudiantiles.

Es importante señalar que para que un docente conciba la equidad, igualdad e inclusión como parte de su quehacer diario en la práctica educativa, es fundamental considerar que:

- Debe fomentar la equidad en el aula y fuera de ella, conviviendo con la comunidad escolar, sin distinción de ningún tipo (raza, edad, sexo, género, cargo o condición social), ya que los estudiantes poseen diferentes formas de aprender, pero el ejemplo y la imitación son las más significativas.

- Los docentes deben hacer diagnósticos en todo momento, que les permitan identificar las necesidades y dificultades que presentan los estudiantes (cognitivas, económicas, sociales, psicoafectivas y de género, entre otras).

- El uso de diferentes estrategias didácticas consideradas para la diversidad en el aula favorece la equidad, principalmente en los procesos de evaluación en donde la arbitrariedad y la injusticia deben eliminarse. 
- Es importante el apoyo a través de tutorías (individuales o colectivas) y / o de asesorías individuales para la atención a necesidades psicoafectivas y académicas.

- Deben considerarse apoyos a requerimientos específicos, como el caso de madres solteras, personas con enfermedades crónicas o dificultades económicas.

- Corresponde al docente romper con los paradigmas existentes en una sociedad discriminante y excluyente.

Actualmente se hace mucho énfasis en la inclusión y las formas de fomentarla en las instituciones educativas, sin embargo, es necesario considerar que se trata de una paradoja, ya que, por un lado, la sociedad y muchas veces las mismas autoridades educativas se encargan de excluir a los individuos y, por otro, «alardean» de los procesos de inclusión. Vemos que los procesos de admisión en las escuelas son excluyentes, que las cuotas de ingreso en ocasiones son tan elevadas que son excluyentes y que las oportunidades que debieran tener todos los individuos no las tienen. Esto es, al observar la desigualdad y la injusticia es imperante la implementación de acciones equitativas para lograr igualdad de oportunidades y de condiciones.

Finalmente es relevante señalar que los formadores de docentes en las Escuelas Normales deben ser un ejemplo para sus propios estudiantes en cuanto al ejercicio de valores y, de manera sustantiva, en cuanto a la equidad. Ello requiere desarrollar colegiadamente programas permanentes de atención a los estudiantes, considerando la organización de la institución para que, desde el plan de desarrollo institucional, se promuevan los procesos equitativos en todos los ámbitos.

El trabajo en las academias, a través de los programas de tutoría y trayectorias estudiantiles que existen en las Escuelas Normales, puede ser el inicio de acciones concretas y permanentes para favorecer la equidad en la formación docente. Ello brindaría a los futuros docentes las condiciones de igualdad, justicia y libertad que debiera poseer todo ser humano.

En relación con las limitaciones del presente estudio, si bien se logró responder al objetivo planteado, la indagación podría ampliarse a fin de obtener una mayor información y comprensión, por ejemplo, de las diferencias entre los profesores que no pertenecen a cuerpos académicos, e incluso contrastar los datos obtenidos con los docentes en formación.

\section{REFERENCIAS}

Amati, M. (s/f). La equidad en la formación docente ¿Deuda pendiente? En file:///C:/ Users/lupit/Downloads/amati.PDF

Bracho-González, T. y Hernández-Fernández, J. (2010). «Equidad educativa: avances en la definición de su concepto. X Congreso Nacional de Investigación Educativa. Veracruz». Memorias, 1-10. En http://www.comie.org.mx/congreso/memoriaelectronica/ $\mathrm{v} 10 / \mathrm{pdf} /$ area tematica $10 /$ ponencias/1852-F.pdf

Canto, R. (2015). «Libertad y diferencia: la contribución del liberalismo igualitario al pensamiento democrático». CONfines de relaciones internacionales y ciencia política, 11(21), 39-61. 
Calvo, G., Camargo, M. y Gutiérrez, M. (2006). Politica educativa, equidad y formación docente. Bogotá, D.C.: Universidad Pedagógica Nacional, Colciencias e Instituto para la Investigación Educativa y el Desarrollo Pedagógico. IDEP

Calvo, G. (2013). «La formación de docentes para la inclusión educativa». Páginas de Educación, 6(1), 19-35. En http:/ / www.scielo.edu.uy/scielo.php?script=sci arttext\&pi$\underline{\mathrm{d}=\mathrm{S} 1688-74682013000100002 \& \operatorname{lng}=\mathrm{es} \& \operatorname{lng}=\mathrm{es}}$.

Cintora, K., González, F. y Vargas, M. (2019). «Formación de tutores inclusivos, una estrategia de inclusión para estudiantes con discapacidad en la Universidad Michoacana de San Nicolás de Hidalgo». XV Congreso Nacional de Investigación Educativa. Ponencia presentada en el Congreso. Acapulco, Guerrero, México.

Compromiso Social (2017). Cómo atiende la SEP la inclusión y la equidad educativa [Formato] Compromiso social por la calidad y la equidad en educación. En https:/ / compromisoporlaeducacion.mx / como-atiende-la-sep-la-inclusion-y-la-equidad-educativa /

Cruz, R. (2019). «Facilitadores y obstaculizadores de la educación inclusiva: análisis desde las experiencias de los profesores de estudiantes con discapacidad». XV Congreso Nacional de Investigación Educativa. Ponencia presentada en el Congreso. Acapulco, Guerrero, México.

Diario Oficial de la Federación (2018). Ley General de Educación. México. En https:// docs.google.com/viewerng/viewer?url=https:/ / legalzone.com.mx/wp-content/ uploads/2018/02/PDF-Ley-General-de-Educaci\%C3\%B3n-legalzone.com .mx . pdf\&hl=es

Escudero, J.M., González, M.T. y Rodríguez, M.J. (2013). «La Mejora Equitativa de la Educación y la Formación del Profesorado». Multidisciplinary Journal of Educational Research, 3(3), 206-234.

Flood, M. (1994). El gasto público social y su impacto. Buenos Aires: Ministerio de Obras y Servicios Públicos de la República de Argentina.

García, I. (2018). «La educación inclusiva en la Reforma Educativa de México». Revista Nacional e Internacional de Educación Inclusiva. Vol. 11, Número 2. En file:/ / C:/Users/ lupit/Downloads / Dialnet-LaEducacionInclusivaEnLaReformaEducativaDeMexico-6729100\%20(1).pdf

Gómez-Robledo, A. (2016). Ética Nicomaquea-Política. México: Porrúa.

Lara-Villanueva, R. (2010). «Las aulas como espacios vivos para construir la equidad escolar». Revista Iberoamericana de Educación. OEI. 51(4). En https://rieoei.org/RIE/ article/view/1821

Martínez, X. y Dijk, S. (2020) «Formación ciudadana y construcción de paz con equidad. Retos actuales de la Educación Media Superior». Revista Panamericana de Pedagogía. «Saberes y Quehaceres del Pedagogo», 29, 59-88.

Martínez, M. (2014) Ciencia y arte en la metodología cualitativa. México: Trillas.

Martínez-Otero, V. (2011). «La mirada a la persona en la educación actual». Revista de Teoría y Didáctica de las Ciencias Sociales, 17, 127-141.

Merino, C. (2018). «Evaluación de tutoría en el Instituto Jaime Torres Bodet: perspectiva del tutorado». En 2do. Congreso Nacional de Investigación sobre Educación Normal. Congreso llevado a cabo en Aguascalientes, Aguascalientes, México. 
Miller, D. y Arvizu, V. (2016). «Ser madre y estudiante. Una exploración de las características de las universitarias con hijos y breves notas para su estudio». Revista de la Educación Superior, XLV(1) (177), 17-42. En https://www.redalyc.org/articulo. oa?id=604/60445151002

Monje-Álvarez, C. (2011). Metodología de la investigación cuantitativa y cualitativa. Guía Didáctica. Neiva. Universidad Surcolombiana.

Organización Internacional del Trabajo [OIT] (2015). Igualdad de oportunidades y de trato. Base de datos sobre legislación nacional del trabajo, la seguridad social y los derechos humanos. En http://www.ilo.org/dyn/natlex/natlex4.detail?p lang=es\&p isn=77170\&p country $=$ MEX\&p count $=712 \& p$ classification $=05 \& p$ classcount $=26 \#: \sim:$ text $=$ Tiene $\% 20$ por $\% 20$ objeto $\% 20$ regular $\% 20 \mathrm{y}, \mathrm{el} \% 20$ empoderamiento $\% 20 \mathrm{de} \% 20 \mathrm{las} \% 20$ mujeres.

Rodríguez-Solera, C. (2008). «Equidad de la Educación en México. Propuesta de un sistema de indicadores». Revista Perspectivas Sociales, 10(2), 55-79. En https:/ / www.uaeh. edu.mx/investigacion/icshu/LI EstuSociales/Carlos Solera/Equidad.pdf

Rawl, J. (2002). La justicia como equidad. Una reformulación. Madrid: Paidós.

Secretaría de Educación Pública [SEP] (2016). El modelo educativo 2016. El planteamiento pedagógico de la Reforma Educativa. Ciudad de México. En http://edu.jalisco.gob.mx/ cepse/sites/edu.jalisco.gob.mx.cepse/files/modelo educativo 2016.pdf

Secretaría de Gobernación (2014). Constitución Política de los Estados Unidos Mexicanos. Vigésima Primera Edición Impresa: Septiembre/2014. En http:// dof.gob.mx/constitucion/marzo 2014 constitucion.pdf

Romo, A. (2011). La tutoría: una estrategia innovadora en el marco de los programas de atención a estudiantes. México: Asociación Nacional de Universidades e Instituciones de Educación Superior, Dirección de Medios Editoriales.

Secretaría de Educación Pública (2002). Licenciatura en Educación Secundaria. Plan de estudios 1999. México: SEP.

Sen, A. (1992). Nuevo examen de la desigualdad. Madrid: Alianza

UNESCO (1990). Declaración mundial sobre educación para todos. Jomtien. UNESCO. En http://www.unesco.org/education/pdf/JOMTIE S.PDF

Valdivielso, S. (coord.) (2016). «Educación y género en la formación docente en un enfoque de equidad y democracia». En el XXXV Seminario Interuniversitario de Teoría de la Educación, bajo el eje temático de la «Democracia y Educación en la Formación Docente». Congreso llevado a cabo en VIC, Barcelona.

Vergara, A. (2005): América Latina: entre luces y sombras. Edición digital accesible a texto completo en http:/ / www.eumed.net/libros/2005/av/ 